\title{
Asymptotics of Varadhan-Type and the Gibbs Variational Principle
}

D. Petz ${ }^{\star}$, G. A. Raggio ${ }^{\star \star}$, and A. Verbeure

Instituut voor Theoretische Fysika, Katholieke Universiteit Leuven, B-3030 Leuven, Belgium

\begin{abstract}
For a large class of quantum models of mean-field type the thermodynamic limit of the free energy density is proved to be given by the Gibbs variational principle. The latter is shown to be equivalent to a noncommutative version of Varadhan's asymptotic formula.
\end{abstract}

\section{Introduction}

Varadhan developed a general theory of the asymptotics of integrals for measures satisfying a large deviation principle [19,9]. An application of this theory generalizes results of Cramér on the rate of convergence in the weak law of large numbers [8]. Let $\xi_{1}, \xi_{2}, \ldots$ be a sequence of independent, identically distributed, random variables, and let $\mu_{n}$ be the distribution of the average

$$
\tilde{x}_{n}=\left(\xi_{1}+\xi_{2}+\ldots+\xi_{n}\right) / n .
$$

Varadhan's result concerns the asymptotic behaviour of the measures $v_{n}$ given by

$$
d v_{n}(u)=\exp (n f(u)) d \mu_{n}(u) .
$$

for a continuous function $f$, and says that

$$
\lim _{n \rightarrow \infty} n^{-1} \log v_{n}(R)=\sup \{f(u)-I(u): u \in R\},
$$

where the rate-function $I$ is determined by the distribution of $\xi_{1}$ [20, Sect. 3].

Here we consider the non-commutative analogue of (1); the random variables become self-adjoint operators in an operator algebra $\mathscr{A}$. To fix the ideas, let $\mathbf{M}$ be the algebra of all complex $m \times m$ matrices and

$$
\mathscr{A}=\bigotimes_{i \in N} \mathbf{M}^{i},
$$

\footnotetext{
* On leave from the Mathematical Institute HAS, Budapest, Hungary

$\star \star$ On leave from the Dublin Institute for Advanced Studies, Dublin, Ireland
} 
where $\mathbf{M}^{i}$ is a copy of $\mathbf{M}$. Let $x=x^{*} \in \mathbf{M}$ and $x_{i}$ be a copy of $x$ in $\mathbf{M}^{i}$. We denote $\left(x_{1}+x_{2}+\ldots+x_{n}\right) / n$ by $\tilde{x}_{n}$. Let $\varrho$ be a faithful state of $M$, and $\omega_{\varrho}$ the associated infinite product-state of $\mathscr{A}$. The sequence $x_{1}, x_{2}, \ldots$ and the state $\omega_{e}$ play the role of independent random variables. We concentrate on the asymptotics of $\operatorname{Tr} \exp \left(\log D_{n}+n f\left(\tilde{x}_{n}\right)\right)$, and compute the limit

$$
\lim _{n \rightarrow \infty} n^{-1} \log \operatorname{Tr} \exp \left(\log D_{n}+n f\left(\tilde{x}_{n}\right)\right)
$$

where $D_{n}$ is the density of $\omega_{\varrho}$ restricted to $\bigotimes_{i=1}^{n} M^{i}$; we show that the limit is equal to

$$
\sup \{f(\varphi(x))-S(\varrho, \varphi): \varphi \in \mathscr{S}\},
$$

where $\mathscr{P}$ is the state space of $\mathbf{M}$, and $S$ is the relative entropy function: $S(\varrho, \varphi)$ $=\operatorname{Tr}\left(D_{\varphi} \log D_{\varphi}\right)-\operatorname{Tr}\left(D_{\varphi} \log D_{\varrho}\right)$, where the $D$ 's are the respective densities. Moreover, we obtain that

$$
\sup \{f(\varphi(x))-S(\varrho, \varphi): \varphi \in \mathscr{S}\}=\sup \left\{f(u)-I_{\varrho, x}(u): u \in R\right\},
$$

where $I_{\varrho, x}$ is the Legendre transform of the function $t \mapsto \log \operatorname{Tr} \exp \left(\log D_{\varrho}+t x\right)$.

These results constitute a non-commutative version of formula (1). Equation (3) relates the rate-function $l$ to the relative entropy. Remark that one recovers the probabilistic situation of $\left[x_{1}, D_{1}\right]=0$. While (1) is derived using measure-theoretic considerations from the large deviation principle:

$$
\begin{array}{ll}
\limsup _{n \rightarrow \infty} n^{-1} \log \mu_{n}[F] \leqq-\inf \{I(u): u \in F\}, & F \text { closed; } \\
\liminf _{n \rightarrow \infty} n^{-1} \log \mu_{n}[G] \geqq-\inf \{I(u): u \in G\}, & G \text { open; }
\end{array}
$$

our proof is different and partially based on a generalization of the de Finetti theorem proved by Størmer [16], which states that any permutation invariant state on an infinite tensor product has a unique decomposition into product states.

From the point of view of statistical mechanics, we establish the existence of the thermodynamic limit of the free-energy density for a large class of quantum meanfield models, and prove that the variational principle is satisfied. Let

$$
D_{\varrho}=\exp (-\beta h) / \operatorname{Tr} \exp (-\beta h),
$$

where $\beta>0$, and $h$ is a self-adjoint element of $\mathbf{M}$, i.e. the Hamiltonian for the single system. The Hamiltonian for $n$ non-interacting systems is then the element $H_{n}^{0}$ of $\bigotimes_{i=1}^{n} \mathbf{M}^{i}$ given by

$$
H_{n}^{0}=\sum_{i=1}^{n} h_{i}
$$

where $h_{i}$ is a copy of $h$ in $\mathbf{M}^{i}$. The mean free energy $F_{n}^{0}(\beta)$ is then simply the free energy of the single system:

$$
F_{n}^{0}(\beta)=(-n \beta)^{-1} \log \operatorname{Tr} \exp \left(-\beta H_{n}^{0}\right)=-\beta^{-1} \log \operatorname{Tr} \exp (-\beta h) .
$$


Suppose the interaction between the systems is of the mean-field type given by $n f\left(\tilde{x}_{n}\right)$, where $x=x^{*} \in \mathbf{M}$; the main example in the physics literature is the quadratic case:

$$
n\left(\tilde{x}_{n}\right)^{2}=(1 / n) \sum_{j, k=1}^{n} x_{j} x_{k},
$$

i.e. the mean-field pair interaction. The Hamiltonian is then

$$
H_{n}=H_{n}^{0}+n f\left(\tilde{x}_{n}\right),
$$

and for the corresponding mean-free energy $F_{n}(\beta)$, one computes that

$$
\beta\left\{F_{n}^{0}(\beta)-F_{n}(\beta)\right\}=n^{-1} \log \operatorname{Tr} \exp \left(\log D_{n}-n \beta f(\tilde{x})\right) .
$$

The existence of the limit of $\left\{F_{n}\right\}$ has been proved before in many particular models (e.g. [7]). Here we prove the existence of the limit for an arbitrary continuous function $f$, and we also allow the single system to be infinite. We prove that the limit is given by the Gibbs variational principle. One should distinguish the existence problem of the thermodynamic limit of the free energy density from the study of the equilibrium conditions for mean fields. In [10] it is shown that the equilibrium condition is equivalent to the famous gap-equation for states.

Finally, we introduce some technical preliminaries. In this paper we consider only unital $C^{*}$-algebras which are inductive limits of finite dimensional $C^{*}$-algebras with a common unit (this class is slightly larger than the class of UHF algebras). The basic ingredient is such a $C^{*}$-algebra $\mathscr{B}$, a fixed selfadjoint element $x$ therein, and a fixed separating state $\varrho$, that is, a state such that the cyclic vector in the GNS construction is separating for the generated von Neumann algebra. Let $\mathscr{A}_{n}$ be the $n$-fold minimal $C^{*}$-tensor-product of $\mathscr{B}$ with itself, and $\mathscr{A}$ the inductive limit $C^{*}$-algebra. If $\varphi$ is a state on $\mathscr{B}$ then $\omega_{\varphi}$ denotes the corresponding infinite product-state on $A$. We remark that if $\varphi$ is separating then also $\omega_{\varphi}$ is [17, Corollary IV.5.12].

Let $\mathbf{M}$ be the von Neumann algebra generated by the GNS representation $\pi$ of $\mathscr{A}$ associated with $\omega_{e}$, and let $\Omega$ be the corresponding cyclic and separating vector. If $h=h^{*} \in \mathscr{A}$, then the perturbed vector $\Omega^{\pi(h)}$ is defined by

$$
\Omega^{\pi(h)}=\exp \left(\frac{\log \Delta+\pi(h)}{2}\right) \Omega,
$$

where $\Delta$ is the modular operator of $(\mathbf{M}, \Omega)$ (see [1]). We define the perturbed functional $\omega_{e}^{h}$ by

$$
\omega_{\varrho}^{h}(a)=\left\langle\pi(a) \Omega^{\pi(h)}, \Omega^{\pi(h)}\right\rangle(a \in A)
$$

(cf. $[4,(5.5)])$.

Every finite permutation $p$ of the positive integers $N$ gives rise to an automorphism $\gamma_{p}$ of $\mathscr{A} ;$ a state of $\mathscr{A}$ is called symmetric if it is invariant under all $\gamma_{p}$ 's. A celebrated theorem of Størmer [16] says that the symmetric states form a simplex $I(\mathscr{A})$ with a closed extreme boundary consisting of the product-states. We shall use this result to reduce the variational problem on the state space of $\mathscr{A}$ to one on the state space of $\mathscr{B}$. 
The proofs are also based on several properties of the relative entropy and the mean relative entropy. For the readers' convenience we collect them in an appendix.

\section{Results}

Let us recall that $\tilde{x}_{n}=\left(x_{1}+x_{2}+\ldots+x_{n}\right) / n$, where $x$ is a fixed self-adjoint element of $\mathscr{B}$. We have:

Theorem 1. Let $\varrho$ be a separating state of the algebra $\mathscr{B}$, and $f$ a continuous realvalued function on the interval $[-\|x\|,\|x\|]$. Then

$$
\lim _{n \rightarrow \infty} n^{-1} \log \left(\omega_{\varrho}\right)^{n f\left(\tilde{x}_{n}\right)}(1)=\sup \left[E_{f}(\omega)-S_{M}\left(\omega_{\varrho}, \omega\right): \omega \in I(\mathscr{A})\right\},
$$

where $E_{f}(\omega)=\lim _{n \rightarrow \infty} \omega\left(f\left(\tilde{x}_{n}\right)\right)$.

The proof is broken up into several lemmas, the first two of which concern the existence of $E_{f}$. We write $C$ for the real-valued continuous functions on $[-\|x\|,\|x\|]$ equipped with the supremum norm.

Lemma 2. For each integer $k \geqq 1$ there exists a constant $c_{k}$ such that for every integer $n \geqq k$, and every $\omega \in I(\mathscr{A})$,

$$
\left|\omega\left(\left(\tilde{x}_{n}\right)^{k}\right)-\omega\left(x_{1} x_{2} \ldots x_{k}\right)\right| \leqq c_{k}\|x\|^{k} / n .
$$

Proof. Using the multinomial expansion and the symmetry,

$$
\begin{aligned}
\omega\left(\left(x_{1}+x_{2}+\ldots+x_{n}\right)^{k}\right)= & (n ! /(n-k) !) \omega\left(x_{1} x_{2} \ldots x_{k}\right) \\
& +\sum\left[k ! / m_{1} ! m_{2} ! \ldots m_{n} !\right] \omega\left(x_{1}^{m_{1}} x_{2}^{m_{2}} \ldots x_{n}^{m_{n}}\right),
\end{aligned}
$$

where the sum is over the non-negative integers $m_{1}, m_{2}, \ldots, m_{n}$ whose sum is $k$, and such that at least one of them is $\geqq 2$, and we have evaluated and summed all summands where every $m_{j}$ is either 0 or 1 . Moreover,

$$
\sum k ! / m_{1} ! m_{2} ! \ldots m_{n} !=n^{k}-(n ! /(n-k) !),
$$

the summation being as before. The claim follows by combining these facts with an estimate by norms. Lemma 3. If $\omega \in I(\mathscr{A})$ and $f \in C$, then $E_{f}(\omega)=\lim _{n \rightarrow \infty} \omega\left(f\left(\tilde{x}_{n}\right)\right)$ exists, and is continuous
in $f$.

Proof. By Lemma 2, $E_{f}$ exists for polynomial $f$, and $\left|E_{f}(\omega)\right| \leqq\|f\|$. The rest follows from the Weierstrass approximation theorem.

Lemma 4. Put $\Phi_{n}=n^{-1} \log \left(\omega_{\varrho}\right)^{n f\left(\tilde{x}_{n}\right)}(1)$, then for every $\omega \in I(\mathscr{A}), \lim \inf \Phi_{n} \geqq E_{f}(\omega)$ $-S_{M}\left(\omega_{\varrho}, \omega\right)$.

Proof. By (A4), $\Phi_{n} \geqq\left(\omega \mid \mathscr{A}_{n}\right)\left(f\left(\tilde{x}_{n}\right)\right)-n^{-1} S\left(\omega_{\varrho}\left|\mathscr{A}_{n}, \omega\right| \mathscr{A}_{n}\right)$. The claim follows from the definition of $S_{M}$ and Lemma 3 .

We have now established that $\liminf _{n \rightarrow \infty} \Phi_{n} \geqq \sup \left\{E_{f}(\omega)-S_{M}\left(\omega_{\varrho}, \omega\right): \omega \in I(\mathscr{A})\right\}$. 
Write $\omega_{n}$ for the state $\left(\omega_{Q} \mid \mathscr{A}_{n}\right)^{n f\left(\tilde{x}_{n}\right)} /\left(\omega_{\varrho} \mid \mathscr{A}_{n}\right)^{n f\left(\tilde{x}_{n}\right)}(1)$ of $\mathscr{A}_{n}$ and remark that $\omega_{n}$ is symmetric. Let $\tilde{\omega}$ be the state of $\mathscr{A}$ defined by

and put

$$
\tilde{\omega}_{n} \mid \mathscr{A}_{k n}=\omega_{n} \otimes \omega_{n} \otimes \ldots \otimes \omega_{n}, \quad \text { all } k \geqq 1,
$$

$$
\bar{\omega}_{n}=n^{-1}\left\{\tilde{\omega}_{n}+\tilde{\omega}_{n} \alpha+\ldots+\tilde{\omega}_{n} \alpha^{n-1}\right\},
$$

where $\alpha$ is the right-shift on $\mathscr{A}$. Notice that $\tilde{\omega}_{n} \alpha^{n}=\tilde{\omega}_{n}$, so that $\bar{\omega}_{n} \alpha=\bar{\omega}_{n}$.

Lemma 5. For integers $n$ and $k$ with $n \geqq k \geqq 1$, and arbitrary elements $a_{1}, a_{2}, \ldots, a_{k}$ in $\mathscr{A}_{1}$,

$$
\left|\left\{\tilde{\omega}_{n}-\bar{\omega}_{n}\right\}\left(a_{1} \alpha\left(a_{2}\right) \ldots \alpha^{k-1}\left(a_{k}\right)\right)\right| \leqq(2(k-1) / n) \prod_{i=1}^{k}\left\|a_{i}\right\| .
$$

Proof.

$$
\begin{aligned}
\bar{\omega}_{n}\left(a_{1} \alpha\left(a_{2}\right) \ldots \alpha^{k-1}\left(a_{k}\right)\right)= & n^{-1} \sum_{j=0}^{n-k} \tilde{\omega}_{n}\left(\alpha^{j}\left(a_{1}\right) \alpha^{j+1}\left(a_{2}\right) \ldots \alpha^{j+k-1}\left(a_{k}\right)\right) \\
& +n^{-1} \sum_{j=n-k+1}^{n-1} \tilde{\omega}_{n}\left(\alpha^{j}\left(a_{1}\right) \alpha^{j+1}\left(a_{2}\right) \ldots \alpha^{j+k-1}\left(a_{k}\right)\right),
\end{aligned}
$$

in the first sum all $(n-k+1)$ summands are equal to $\tilde{\omega}_{n}\left(a_{1} \alpha\left(a_{2}\right) \ldots \alpha^{k-1}\left(a_{k}\right)\right)$ $=\omega_{n}\left(a_{1} \alpha\left(a_{2}\right) \ldots \alpha^{k-1}\left(a_{k}\right)\right)$ since $j+k \leqq n$; the modulus of the second sum is estimated by $(n(n-k+1)) \prod_{i=1}^{k}\left\|a_{i}\right\|$.

Remark that this implies that $\left\{\tilde{\omega}_{n}\right\}$, and $\left\{\bar{\omega}_{n}\right\}$ have the same limit points, which are symmetric.

The following is the main estimate for the energy part.

Lemma 6. If $N_{0}$ is an inf inite subset of the positive integers, $\omega$ is a $w^{*}$-limit point of $\left\{\bar{\omega}_{n}: n \in N_{0}\right\}$, and $f \in C$, then given $\varepsilon>0,\left|E_{f}(\omega)-\omega_{n}\left(f\left(\tilde{x}_{n}\right)\right)\right| \leqq \varepsilon$, for infinitely many in $N_{0}$.

Proof. $\omega$ is symmetric so that $E_{f}(\omega)$ is given by Lemma 3. If $f$ is a polynomial,

$$
f(u)=\sum_{k=0}^{l} c_{k} u^{k}
$$

then using Lemma 5 choose $n_{0}$ sufficiently large so that

$$
\left|\sum_{k=0}^{l} c_{k}\left(\tilde{\omega}_{n}\left(x_{1} x_{2} \ldots x_{k}\right)-\bar{\omega}_{n}\left(x_{1} x_{2} \ldots x_{k}\right)\right)\right| \leqq \varepsilon, \quad \text { for all } n \geqq n_{0},
$$
with the understanding that $x_{0}=1$. Since $E_{f}(\omega)=\sum_{k=0}^{l} c_{k} \omega\left(x_{1} x_{2} \ldots x_{k}\right)$, we can
choose an infinite subset $N_{1}$ of $N_{0}$ such that

$$
\left|\sum_{k=0}^{l} c_{k}\left(\omega\left(x_{1} x_{2} \ldots x_{k}\right)-\bar{\omega}_{n}\left(x_{1} x_{2} \ldots x_{k}\right)\right)\right| \leqq \varepsilon, \quad \text { for all } n \in N_{1} \text {. }
$$

Finally, notice that for $n$ sufficiently large, $\omega_{n}\left(x_{1} x_{2} \ldots x_{k}\right)=\tilde{\omega}_{n}\left(x_{1} x_{2} \ldots x_{k}\right)$, so that the claim follows for polynomial $f$. For general $f \in C$, we obtain the result by the continuity of the map $f \mapsto E_{f}(\omega)$ established in Lemma 3 . 
We now turn to the entropy estimates. In the following two lemmas $n$ is an arbitrary positive integer.

Lemma 7. For every positive integer $j$,

$$
\lim _{k \rightarrow \infty} k^{-1} S\left(\omega_{g}\left|\mathscr{A}_{k}, \tilde{\omega}_{n} \alpha^{j}\right| \mathscr{A}_{k}\right)=n^{-1} S\left(\omega_{g} \mid \mathscr{A}_{n}, \omega_{n}\right)
$$

Proof. Using the $\alpha^{n}$-invariance of $\tilde{\omega}_{n}$, we may assume that $0 \leqq j<n$. For $k \geqq n-j$, put $k=n-j+m n+k_{0}$, where $0 \leqq k_{0}<n$, and $m$ is an integer. Then,

$$
\tilde{\omega}_{n} \alpha^{j}=\left(\omega_{n} \mid A_{n-j}\right) \otimes \omega_{n} \otimes \omega_{n} \otimes \ldots,
$$

so that

$$
\begin{aligned}
S\left(\omega_{\varrho}\left|\mathscr{A}_{k}, \tilde{\omega}_{n} \alpha^{j}\right| \mathscr{A}_{k}\right)= & m S\left(\omega_{p} \mid \mathscr{A}_{n}, \omega_{n}\right) \\
& +S\left(\omega_{\varrho}\left|\mathscr{A}_{n-j}, \omega_{n}\right| \mathscr{A}_{n-j}\right)+S\left(\omega_{\varrho}\left|\mathscr{A}_{k_{0}}, \omega_{n}\right| \mathscr{A}_{k_{0}}\right) .
\end{aligned}
$$

Since the last two summands are bounded by $S\left(\omega_{\varrho} \mid \mathscr{A}_{n}, \omega_{n}\right)$ due to monotonicity of the relative entropy, the claim follows by dividing by $k$ and taking the limit $m \rightarrow \infty$.

Lemma 8. $S_{M}\left(\omega_{\varrho}, \bar{\omega}_{n}\right)=n^{-1} S\left(\omega_{\varrho} \mid \mathscr{A}_{n}, \omega_{n}\right)$.

Proof. We have $S_{M}\left(\omega_{\varrho}, \bar{\omega}_{n}\right)=n^{-1} S_{M}^{n}\left(\omega_{\varrho}, \bar{\omega}_{n}\right)$, by scaling. Since each $\tilde{\omega}_{n} \cdot \alpha^{j}$ $(j=0,1, \ldots, n)$ is a $\alpha^{n}$-invariant, the affinity of the mean relative entropy (A5) implies

$$
\left.S_{M}^{n}\left(\omega_{\varrho}, \omega_{n}\right)=n^{-1} \sum_{j=0}^{n-1} S_{M}^{n}\left(\omega_{\varrho}, \tilde{\omega}_{n} \alpha^{j}\right)\right)
$$

Thus, by the definition of $S_{M}^{n}$,

$$
S_{M}\left(\omega_{\varrho}, \bar{\omega}_{n}\right)=n^{-2} \sum_{j=0}^{n-1} \lim _{k \rightarrow \infty} k^{-1} S\left(\omega_{\varrho}\left|\mathscr{A}_{k n}, \tilde{\omega}_{n} \alpha^{j}\right| \mathscr{A}_{k n}\right)
$$

The claim follows from Lemma 7.

We can now give the proof of Theorem 1. By the remark after Lemma 4 it suffices to show that given $\varepsilon>0$,

$$
c \equiv \limsup _{n \rightarrow \infty} \Phi_{n} \leqq E_{f}(\varphi)-S_{M}\left(\omega_{\varrho}, \varphi\right)+\varepsilon,
$$

for some $\varphi \in I(\mathscr{A})$. There is an infinite subset $N_{0}$ of the integers such that

$$
\omega_{n}\left(f\left(\tilde{x}_{n}\right)\right)-n^{-1} S\left(\omega_{0} \mid \mathscr{A}_{n}, \omega_{n}\right)=\Phi_{n} \geqq c-\varepsilon, \quad \text { for all } n \in \mathbf{N}_{0},
$$

where we have used (A4); let $\omega$ ) be a $w^{*}$-limit point of $\left\{\bar{\omega}_{n}: n \in \mathbf{N}_{0}\right\}$; by Lemma 6 there is an infinite subset $\mathbf{N}_{1}$ of $\mathbf{N}_{0}$ such that

$$
E_{f}(\omega) \geqq \omega_{n}\left(f\left(\tilde{x}_{n}\right)\right)-\varepsilon, \quad \text { for all } n \in \mathbf{N}_{1} .
$$

By the lower semicontinuity (A5) of $S_{M}\left(\omega_{\varrho}, \omega\right)$

$$
S_{M}\left(\omega_{\varrho}, \omega\right) \leqq S_{M}\left(\omega_{\varrho}, \bar{\omega}_{n_{1}}\right)+\varepsilon
$$


for some $n_{1} \in \mathbf{N}_{n}$. Due to Lemma 8 , we then have

$$
\begin{aligned}
E_{f}(\omega)-S_{M}\left(\omega_{\varrho}, \omega\right) & \geqq \omega_{n_{1}}\left(f\left(\tilde{x}_{n_{1}}\right)\right)-S_{M}\left(\omega_{\varrho}, \bar{\omega}_{n_{1}}\right)-2 \varepsilon \\
& =\omega_{n_{1}}\left(f\left(\tilde{x}_{n_{1}}\right)\right)-n_{1}^{-1} S\left(\omega_{\varrho} \mid A_{n_{1}}, \omega_{n_{1}}\right)-2 \varepsilon \geqq c-3 \varepsilon .
\end{aligned}
$$

This completes the proof of Theorem 1.

The argument of the proof of Theorem 1 can be repeated to prove that every $w^{*}$-limit point of $\left\{\omega_{\varrho}^{n f\left(\tilde{x}_{n}\right)} / \omega_{\varrho}^{n f\left(\tilde{x}_{n}\right)}(1): n \in \mathbf{N}\right\}$ is a maximizer of $E_{f}(\cdot)-S_{M}\left(\omega_{\varrho}, \cdot\right)$.

Let the real-valued function $G$ on the reals be defined by

$$
G(t)=\log \varrho^{t x}(1) .
$$

Obviously,

$$
G^{\prime}(t)=\varrho^{t x}(x) / \varrho^{t x}(1) .
$$

and $G^{\prime \prime}(t)>0$ if $x$ is not a multiple of the identity (see Lemma 1 of [2] for $t=0$, and note that due to the chain rule $\varrho^{(t+s) x}=\left(\left(\varrho^{t x}\right)^{s x}\right.$, the general case is similar $)$. Since $G^{\prime}$ is non-decreasing, we have

$$
\begin{aligned}
& z_{+}=\sup \left\{G^{\prime}(t): t \in R\right\}=\lim _{t \rightarrow \infty} G^{\prime}(t) \leqq\|x\|, \\
& z_{-}=\inf \left\{G^{\prime}(t): t \in R\right\}=\lim _{t \rightarrow \infty} G^{\prime}(t) \geqq-\|x\|,
\end{aligned}
$$

with $z_{-}<z_{+}$if $x$ is not a multiple of the identity. We assume in what follows that this is the case. The proof of the following lemma is straightforward; we leave it to the reader.

Lemma 9. Let $I(u)=\sup \{t u-G(t)\}, u \in R$, be the Legendre transform of $G$. I is nonnegative with $l(\varrho(x))=0$, and $l(u)=+\infty$ if $u$ is outside the interval $\left(z_{-}, z_{+}\right)$. Moreover, $l(u)$ is finite if $G^{\prime}(t)=u$ has a solution, and then $l(u)=t u-G(t)$.

Lemma 10. For each $\varphi \in \mathscr{S}, S(\varrho, \varphi) \geqq I(\varphi(x))$. If $S(\varrho, \varphi)=I(\varphi(x))<\infty$ and $I\left(z_{+}\right)$ $=I\left(z_{-}\right)=\infty$, then there is a real $t$ such that $\varphi=\varrho^{t x} / Q^{t x}(1)$ and conversely.

Proof. We use (A4). $S(\varrho, \varphi) \geqq \sup \left\{\varphi(h)-\log \varrho^{h}(1): h^{*}=h \in \mathscr{B}\right\} \geqq \sup \quad\{t \varphi(x)$ $-G(t): t \in R\}=I(\varphi(x))$. If $\varphi=\varrho^{t x} / \varrho^{t x}(1)$, then $S(\varrho, \varphi)=t \varphi(x)-G(t) \leqq I(\varphi(x))$. Suppose $S(\varrho, \varphi)=I(\varphi(x))<\infty$; if $x$ is a multiple of the identity then $I(\varphi(x))=0$, which implies $\varrho=\varphi$; otherwise, by strict convexity of $G, I(\varphi(x))=t_{0} \varphi(x)-G\left(t_{0}\right)$, where $t_{0}$ is the unique solution of $\varphi(x)=G^{\prime}(t)$, and $\varphi=\varrho^{t x} / \varrho^{t x}(1)$.

Lemma 11. One has

$$
\begin{aligned}
\sup \{(f(u)-I(u): u \in[-\|x\|,\|x\|]\} & =\sup \left\{f\left(G^{\prime}(t)\right)-t G^{\prime}(t)+G(t): t \in R\right\} \\
& =\sup \{f(\varphi(x))-S(\varrho, \varphi): \varphi \in \mathscr{S}\} .
\end{aligned}
$$

If $I\left(z_{+}\right)=I\left(z_{-}\right)=\infty$ then the sets of maximizers are in one-to-one correspondence as follows:

(i) Given a miximizing $u$, $t$ is the unique solution of $G^{\prime}(t)=u$, and $\varphi=\varrho^{t x} / \varrho^{t x}(1)$;

(ii) Given a maximizing $t, u=G^{\prime}(t)$, and $\varphi=\varrho^{t x} / \varrho^{t x}(1)$;

(iii) Given a maximizing $\varphi, u=\varphi(x)$, and $t$ is the unique solution of $G^{\prime}(t)=\varphi(x)$. 
Proof. The case where $x$ is a multiple of the identitiy trivializes. Suppose this is not the case; by Lemma 10,

$$
\begin{aligned}
\sup \{f(\varphi(x))-S(\varrho, \varphi): \varphi \in \mathscr{S}\} & \leqq \sup \{f(\varphi(x))-I(\varphi(x)): \varphi \in \mathscr{S}\} \\
& \leqq \sup \{f(u)-I(u): u \in[-\|x\|,\|x\|]\}=s .
\end{aligned}
$$

Let $u \in\left(z_{-}, z_{+}\right)$. By the strict convexity of $G$, there exists a unique real $t_{u}$ such that

$$
u=G^{\prime}\left(t_{u}\right) \text {. }
$$

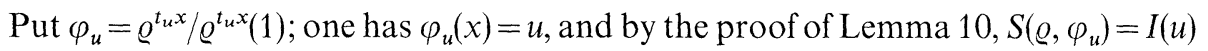
$=t_{u} G^{\prime}\left(t_{u}\right)-G\left(t_{u}\right)$. Now,

$$
\begin{aligned}
\sup \{f(\varphi(x))-S(\varrho, \varphi): \varphi \in \mathscr{S}\} & \geqq \sup \left\{f\left(\varphi_{u}(x)\right)-S\left(\varrho, \varphi_{u}\right): z_{-}<u<z_{+}\right\} \\
& =\sup \left\{f(u)-I(u): z_{-}<u<z_{+}\right\},
\end{aligned}
$$

and, by the upper semicontinuity of $f(u)-I(u)$ the last supremum is equal to $s$. But, also $f\left(\varphi_{u}(x)\right)-S\left(\varrho, \varphi_{u}\right)=f\left(G^{\prime}\left(t_{u}\right)\right)-t_{u} G^{\prime}\left(t_{u}\right)+G\left(t_{u}\right)$, and as $u$ varies in $\left(z_{-}, z_{+}\right), t$ varies in $R$. The supremum over $u \in[-\|x\|,\|x\|]$ is attained (in $\left[z_{-}, z_{+}\right]$) by continuity. The last claim follows using Lemma 10.

The main result establishes the bridge between the Gibbs variational principle and an asymptotic formula of the type given by Varadhan (cf. [9, pp. 50-51]).

Theorem 12. Let $\varrho$ be a separating state of the algebra $\mathscr{B}$, and $f$ a continuous realvalued function on the interval $[-\|x\|,\|x\|]$. Then

$$
\begin{aligned}
& \lim _{n \rightarrow \infty} n^{-1} \log \left(\omega_{\varrho}\right)^{n f\left(\tilde{x}_{n}\right)}(1) \sup \{f(\varphi(x))-S(\varrho, \varphi): \varphi \text { a state on } \mathscr{B}\} \\
& \quad=\sup \{f(u)-I(u): u \in[-\|x\|,\|x\|]\} .
\end{aligned}
$$

Moreover, if $\omega$ is a $w^{*}$-limit point of the sequence $\left\{\omega_{n}\right\}$, then there is a regular probability measure $\mu_{\omega}$ on the state space $\mathscr{S}$ of $\mathscr{B}$ such that

$$
\omega=\int \omega_{\varphi} d \mu_{\omega}(\varphi)
$$

and $\mu_{\omega}$ is supported by the states $\varphi \in \mathscr{S}$ maximizing the first supremum.

Proof. For $\varphi \in \mathscr{S}$, and a polynomial $f$, we see from Lemma 2 that

$$
E_{f}\left(\omega_{\varphi}\right)=f(\varphi(x))
$$

this formula extends by continuity (Lemma 3) to every $f \in C$. Since $S_{M}\left(\omega_{\underline{Q}}, \omega_{\varphi}\right)$ $=S(\varrho, \varphi)$, we have

$$
\sup \left\{E_{f}(\omega)-S_{M}\left(\omega_{\varrho}, \omega\right): \omega \in I(A)\right\} \geqq \sup \{f(\varphi(x))-S(\varrho, \varphi): \varphi \in \mathscr{S}\} .
$$

Given $\omega \in I(\mathscr{A})$, there exists a unique regular probability measure $\mu_{\omega}$ on $\mathscr{S}[16]$, such that

$$
\omega=\int \omega_{\varphi} d \mu_{\omega}(\varphi)
$$

If $f$ is a polynomial, then by Lemma 2 ,

$$
E_{f}(\omega)=\int f(\varphi(x)) d \mu_{\omega}(\varphi)
$$


and this formula extends by continuity to $C$. Moreover, the affinity and lower semicontinuity of the mean relative entropy (A5) imply [13, Lemma 9.7, p. 68]

$$
S_{M}\left(\omega_{\varrho}, \omega\right)=\int S_{M}\left(\omega_{\varrho}, \omega_{\varphi}\right) d \mu_{\omega}(\varphi)=\int S(\varrho, \varphi) d \mu_{\omega}(\varphi) .
$$

Let $\omega \in I(\mathscr{A})$ maximize $E_{f}(\cdot)-S_{M}\left(\omega_{\varrho}\right)$. Then

$$
\begin{aligned}
E_{f}(\omega)-S_{M}\left(\omega_{\varrho}, \omega\right) & =\int\{f(\varphi(x))-S(\varrho, \varphi)\} d \mu_{\omega}(\varphi) \\
& \leqq \sup \left\{f(\varphi(x))-S(\varrho, \varphi): \varphi \in \operatorname{supp}\left(\mu_{\omega}\right)\right\} .
\end{aligned}
$$

Together with Theorem 1 and Lemma 11 this gives the first claim.

As noted after the proof of Theorem 1, any $w^{*}$-limit point of $\left\{\omega_{n}\right\}$ maximizes the function $E_{f}(\cdot)-S_{M}\left(\omega_{\varrho}, \cdot\right)$ on the state space of $\mathscr{A}$; therefore, for such a limit point $\omega, \varphi$ maximizes $f(\cdot(x))-S(\varrho, \cdot)$ for $\mu_{\omega}$ - almost all $\varphi \in \mathscr{S}$. Due to the upper semicontinuity of $\varphi \mapsto f(\varphi(x))-S(\varrho, \varphi)$ [see (A2)], the set of its maximizers is closed.

Although the primary aim of the present paper was to prove the existence of $\lim \Phi_{n}$, and establish the connection with Varadhan's asymptotic formula, we $n \rightarrow \infty$
obtain as a byproduct, the so-called gap-equation.

Assume that $f$ is twice continuously differentiable, and let $\omega=\int \omega_{\varphi} d \mu_{\omega}(\varphi)$ be as in the previous theorem. Then, for $\mu_{\omega}$-almost all $\varphi \in \mathscr{S}$ there exists $t(\varphi) \in R$ such that
(i) $\varphi=\varrho^{t(\varphi) x} / \varrho^{t(\varphi) x}(1)$,
(ii) $f^{\prime}\left(G^{\prime}(t(\varphi))=t(\varphi)\right.$,
(iii) $f^{\prime \prime}\left(G^{\prime}(t(\varphi))\right)<1 / G^{\prime \prime}(t(\varphi))$.

Set $F(t)=f\left(G^{\prime}(t)\right)-t G^{\prime}(t)+G(t)$. One computes that

$$
\begin{gathered}
F^{\prime}(t)=G^{\prime \prime}(t)\left[f^{\prime}\left(G^{\prime}(t)\right)-t\right], \\
F^{\prime \prime}(t)=G^{\prime \prime}(t)\left[f^{\prime \prime}\left(G^{\prime}(t)\right) G^{\prime \prime}(t)-1\right]+G^{\prime \prime \prime}(t)\left[f^{\prime}\left(G^{\prime}(t)\right)-t\right] .
\end{gathered}
$$

Lemma 11 tells is that the maximizers of the functional $\varphi \mapsto f(\varphi(x))-S(\varrho, \varphi)$, are in one-to-one correspondence with the maximizers of $t \mapsto F(t)$. Since $\operatorname{sgn} F^{\prime}(t)=-\operatorname{sgn} t$ if $|t|$ is large enough, the maxima are attained at those $t$ satisfying $F^{\prime}(t)=0$ and $F^{\prime \prime}(t)<0$. So we arrive at (ii) and (iii) since $G^{\prime \prime}(t)>0$.

The condition (ii) is the well-known gap-equation of the corresponding mean field model [10, Theorem II.4]. On the other hand, condition (iii) selects the thermodynamically stable solutions of (ii) (cf. [14, Eq. (8)]).

\section{Appendix on Entropies}

Let $\mathbf{M}$ be a finite dimensional $C^{*}$-algebra with a faithful tracial state $\tau$. To every positive functional $\varphi$ on $M$ there corresponds a density $D_{\varphi}$ with respect to $\tau$. If $\varphi$ is faithful, $D_{\varphi}$ is invertible and the quantity

$$
S(\varphi, \omega)=\tau\left(D_{\omega} \log D_{\omega}\right)-\tau\left(D_{\omega} \log D_{\varphi}\right)
$$


is independent of $\tau$. It is called the relative entropy of $\varphi$ and $\omega$ ([18], see also [11]). The following properties of the relative entropy are known (see, e.g., [6, pp. 269-305]):

I. Scaling: For $\lambda, \mu>0, S(\lambda \varphi, \mu \omega)=\mu S(\varphi, \omega)+\omega(1) \mu \log (\mu / \lambda)$;

II. Convexity: For $0<\lambda<1$,

$$
\begin{aligned}
& \lambda S\left(\varphi, \omega_{1}\right)+(1-\lambda) S\left(\varphi, \omega_{2}\right)+\lambda \log \lambda+(1-\lambda) \log (1-\lambda) \\
& \quad \leqq S\left(\varphi, \lambda \omega_{1}+(1-\lambda) \omega_{2}\right) \leqq \lambda S\left(\varphi, \omega_{1}\right)+(1-\lambda) S\left(\varphi, \omega_{2}\right) ;
\end{aligned}
$$

III. Superadditivity: if $\mathbf{M}=\mathbf{M}_{1} \otimes \mathbf{M}_{2} \varphi=\varphi_{1} \otimes \varphi_{2}$, and $\omega$ restricted to $\mathbf{M}_{i}$ is denoted by $\omega_{i}(i=1,2)$, then

$$
S(\varphi, \omega) \geqq S\left(\varphi_{1}, \omega_{1}\right)+S\left(\varphi_{2}, \omega_{2}\right) ;
$$

IV. Monotonicity: If $\mathbf{N} \subseteq \mathbf{M}$ then

$$
S(\varphi, \omega) \geqq S(\varphi|\mathbf{N}, \omega| \mathbf{N}) \geqq \omega(1)[\log \omega(1)-\log \varphi(1)] .
$$

Araki [4] extended the definition of the relative entropy to normal states of an arbitrary von Neumann algebra; the extension has all the above properties.

In the following four propositions $\mathscr{A}$ is a unital $C^{*}$-algebra which is the inductive limit of finite-dimensional $C^{*}$-algebras and $\varphi$ is a fixed separating state of $\mathscr{A}$. The GNS triplet associated with $\varphi$ is written $\left(\pi_{\varphi}, H_{\varphi}, \Phi\right)$. There exists an increasing sequence $\left\{\mathbf{M}_{n}\right\}$ of finite-dimensional subalgebras $\mathbf{M}_{n}$ of $\mathscr{A}$ such that $\bigcup_{n \in N} \mathbf{M}_{n}$ is dense in $\mathscr{A}$. If $\omega$ is an arbitrary state of $\mathscr{A}$, we define

$$
S(\varphi, \omega)=\lim _{n \rightarrow \infty} S\left(\varphi\left|M_{n}, \omega\right| M_{n}\right) .
$$

The limit exists due to monotonicity and is non-negative or $+\infty$.

A1. Proposition. If $\sup \left\{S\left(\varphi\left|\mathbf{M}_{n}, \omega\right| \mathbf{M}_{n}\right): n \in N\right\}$ is finite, then there exists a vector $\Omega \in H_{\varphi}$ such that $\omega(a)=\left\langle\pi_{\varphi}(a) \Omega, \Omega\right\rangle(a \in \mathscr{A})$.

This proposition follows immediately from Sect. 5 of [4], where it is shown that under the present conditions $\pi_{\varphi}$ quasi-contains $\pi_{\omega}$.

Using the above proposition, and the martingale property [4, Theorem 3.9] of the relative entropy of normal states of a von Neumann algebra, we obtain an equivalent definition of $S(\varphi, \omega)$. Namely, $S(\varphi, \omega)=+\infty$, if $\omega$ does not admit a normal extension to a state of the von Neumann algebra $\pi_{\varphi}(\mathscr{A})^{\prime \prime}$; if such an extension (unique) $\tilde{\omega}$ exists, then

$$
S(\varphi, \omega)=S(\tilde{\varphi}, \tilde{\omega})
$$

where $\tilde{\varphi}$ is the vector state given by $\Phi$, and the quantity on the right-hand side is the relative entropy of the normal states $\tilde{\varphi}$ and $\tilde{\omega}$ (cf. [4, Sect. 5]).

A2. Proposition. $S(\varphi, \omega)$ is a $w^{*}$ lower semicontinuous function of $\omega$.

Proof. Due to the monotonicity, $S(\varphi, \omega)=\sup _{n \in N} S\left(\varphi\left|\mathbf{M}_{n}, \omega\right| \mathbf{M}_{n}\right)$, and $S\left(\varphi\left|\mathbf{M}_{n}, \omega\right| \mathbf{M}_{n}\right)$ is continuous in $\omega$ because $\mathbf{M}_{n}$ is finite-dimensional. 
A3. Proposition. If $h=h^{*} \in \mathscr{A}$, then $S\left(\varphi, \varphi^{h}\right)=\varphi^{h}(h)$.

Proof. This is immediate from the corresponding result for normal states of a von Neumann algebra [4, Theorem 3.10].

A4. Proposition. If $h=h^{*} \in \mathscr{A}$, then $\log \varphi^{h}(1) \geqq \omega(h)-S(\varphi, \omega)$ for every state $\omega$ on $\mathscr{A}$, and equality holds only when $\omega=\varphi^{h} / \varphi^{h}(1)$.

Proof. If $S(\varphi, \omega)=+\infty$ there is nothing to prove. Otherwise, applying A1 reduces the problem to that for normal states of a von Neumann algebra. Let $\tilde{\varphi}$ and $\tilde{\omega}$ be the normal state extensions of $\varphi$ and $\omega$ to $\pi_{\varphi}(A)^{\prime \prime}$. Then $\log \varphi^{h}(1)=\log \tilde{\varphi}^{\pi} \varphi^{(h)}(1)$, and $\omega(h)-S(\varphi, \omega)=\tilde{\omega}\left(\pi_{\varphi}(h)\right)-S(\tilde{\varphi}, \tilde{\omega})$. Since

$$
S(\tilde{\varphi}, \tilde{\omega})-\tilde{\omega}\left(\pi_{\varphi}(h)\right)=S\left(\tilde{\varphi}^{\pi_{\varphi}(h)}, \omega\right)
$$

(see [4]) the monotonicity $S\left(\tilde{\varphi}^{\pi_{\varphi}(h)}, \tilde{\omega}\right) \geqq \tilde{\omega}(1)\left[\log \tilde{\omega}(1)-\log \tilde{\varphi}^{\pi_{\varphi}(h)}(1)\right]$ gives the inequality.

If $\omega=\varphi^{h} / \varphi^{h}(1)$, then $S(\varphi, \omega)=\left\{\varphi^{h} / \varphi^{h}(1)\right\}(h)+\log \varphi^{h}(1)$, by A3 and I.

Now suppose equality holds true; we proceed as in the proof of Proposition 1 of [12], even if we do not know a priori that $\omega$ is faithful. The equality implies

$$
p\left[D \tilde{\varphi}^{\pi_{\varphi}(h)}, D \tilde{\omega}\right]_{t} p=\left\{\varphi^{h}(1) / \omega(1)\right\}^{i t} p .
$$

where $p$ is the support-projection of $\tilde{\omega}$. Since $\left[D \tilde{\varphi}^{\pi_{\varphi}(h)}, D \tilde{\omega}\right]_{t}$ is a partial isometry from $p$ into $p$, we have, introducing the notation $\tilde{\mu}=\tilde{\varphi}^{\pi_{\varphi}(h)} / \varphi^{h}(1)$,

$$
[D \tilde{\mu}, D \tilde{\omega}]_{t}=p .
$$

This implies (see $[5$, Theorem B.1]) that $\tilde{\omega}=p \tilde{\mu} p$. Then $\tilde{\mu}(p)=1$, and we arrive at $p=1$, and $\tilde{\omega}=\tilde{\varphi}^{\pi_{\varphi}(h)} / \varphi^{h}(1)$.

We return to the case where $\mathscr{A}_{n}$ (respectively $\mathscr{A}$ ) is the $n$-fold (respectively infinite) tensor product of $\mathscr{B}$, and $\varrho$ is a separating state of $\mathscr{B}$. For a state $\omega$ of $\mathscr{A}$ invariant under $\alpha^{k}(k \in N)$ we have

$$
S\left(\omega_{\varrho}\left|\mathscr{A}_{k(n+m)}, \omega\right| \mathscr{A}_{k(n+m)}\right) \geqq S\left(\omega_{\varrho}\left|\mathscr{A}_{k n}, \omega\right| \mathscr{A}_{k n}\right)+S\left(\omega_{\varrho}\left|\mathscr{A}_{k m}, \omega\right| \mathscr{A}_{k m}\right)
$$

as a consequence of superadditivity. Therefore [9, Lemma IX.2.5, p. 274]

$$
\lim _{n \rightarrow \infty} n^{-1} S\left(\omega_{\varrho}\left|\mathscr{A}_{k n}, \omega\right| \mathscr{A}_{k n}\right)=\sup _{n \in N} n^{-1} S\left(\omega_{\varrho}\left|\mathscr{A}_{k n}, \omega\right| \mathscr{A}_{k n}\right),
$$

and we call this number the $k$-step mean relative entropy $S_{M}^{k}\left(\omega_{\varrho}, \omega\right)$ of $\omega_{\varrho}$ and $\omega$. Instead of $S_{M}^{1}$ we write $S_{M}$ and call it the mean relative entropy.

A5. Proposition. $S_{M}^{k}\left(\omega_{\varrho}, \omega\right)$ is a $w^{*}$ lower semicontinuous, affine function of the $\alpha^{k}$-invariant state $\omega$. Moreover, $S_{M}^{k}\left(\omega_{\varrho}, \omega\right)=k S_{M}\left(\omega_{\varrho}, \omega\right)$ if $\omega$ is $\alpha$-invariant.

Proof. The lower semicontinuity follows from A2. Affinity follows from the convexity (II). The scaling property is obvious. 


\section{References}

1. Araki, H.: Relative hamiltonian for faithful normal states of a von Neumann algebra. Publ. Res. Inst. Math. Sci. 9, 165-209 (1973)

2. Araki, H.: Golden-Thompson and Peierls-Bogoliubov inequalities for a general von Neumann algebra. Commun. Math. Phys. 34, 167-178 (1973)

3. Araki, H.: On uniqueness of KMS states of one-dimensional quantum lattice systems. Commun. Math. Phys. 44, 1-7 (1975)

4. Araki, H.: Relative entropy of states of von Neumann algebras. II. Publ. Res. Inst. Math. Sci. 13, 173-192 (1977)

5. Araki, H., Masuda, T.: Positive cones and $L_{p}$-spaces for von Neumann algebras. Publ. Res. Inst. Math. Sci. 18, 339-411 (1982)

6. Bratteli, O., Robinson, D.W.: Operator algebras and quantum statistical mechanics. II. Berlin, Heidelberg, New York: Springer 1981

7. Cegła, W., Lewis, J.T., Raggio, G.A.: The free energy of quantum spin systems and large deviations. Commun. Math. Phys. 118, 337-354 (1988)

8. Cramér, H.: On a new limit theorem in the theory of probability. In: Colloquium on the theory of probability. Paris: Hermann 1937

9. Ellis, R.S.: Entropy, large deviations and statistical mechanics. Berlin, Heidelberg, New York: Springer 1985

10. Fannes, M., Spohn, H., Verbeuere, A.: Equilibrium states for mean field models. J. Math. Phys. 21, 355-358 (1980)

11. Petz, D.: Quasi-entropies for finite quantum systems. Rep. Math. Phys. 23, 57-65 (1986)

12. Petz, D.: A variational expression for the relative entropy. Commun. Math. Phys. 114, 345-349 (1988)

13. Phelps, R.R.: Lectures on Choquet's theorem. New York, Toronto, London, Melbourne: Van Nostrand 1966

14. Quaegebeur, J., Verbeuere, A.: Stability for mean field models. Ann. Inst. Henri Poincaré A 22, 343-349 (1980)

15. Segal, I.E., Kunze, R.A.: Integrals and operators. Berlin, Heidelberg, New York: Springer 1978

16. Størmer, E.: Symmetric states of infinite tensor products of $C^{*}$-algebras. J. Funct. Anal. 3, 48-68 (1969)

17. Takesaki, M.: Theory of operator algebras I. Berlin, Heidelberg, New York: Springer 1979

18. Umegaki, H.: Conditional expectations in an operator algebra IV (Entropy and information). Kodai Math. Sem. Rep. 14, 59-85 (1962)

19. Varadhan, S.R.S.: Asymptotic probabilities and differential equations. Commun. Pure Appl. Math. 19, 261-286 (1966)

20. Varadhan, S.R.S.: Large deviations and applications. Philadelphia, PA: Society for Industrial and Applied Mathematics 1984

Communicated by H. Araki

Received July 4, 1988; in revised form October 1, 1988 